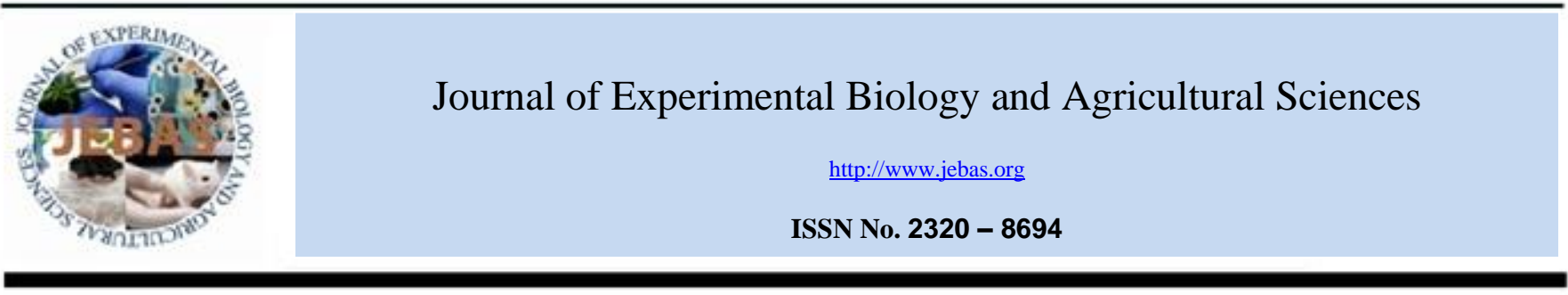

\title{
ASSESSMENT OF THE ANTIOXIDANT PROPERTIES OF THE MOST COMMON COFFEE BREWS AVAILABLE IN THE LOCAL MARKETS OF THE WESTERN REGION OF SAUDI ARABIA
}

\author{
Huda A. Al Doghaither*, Ashjan M. Almowalad, Ayat M. Shorbaji, Ayat B. Al-Ghafari, and \\ Ulfat M. Omar
}

Biochemistry Department, Faculty of Sciences, King Abdulaziz University, Jeddah, Kingdom of Saudi Arabia

Received - January 11, 2017; Revision- January 20, 2017; Accepted - February 20, 2017

Available Online - February 28, 2017

DOI: http://dx.doi.org/10.18006/2017.5(1).070.076

\begin{abstract}
KEYWORDS
Antioxidants

Coffee

Phenolic

Flavonoid

DPPH

Metal chelation

ABSTRACT

The purpose of the current study is to determine the antioxidant activity of the most commonly used coffee brews in the Western region of Saudi Arabia. Further, total phenolic and flavonoid contents were measured and antioxidants properties including ferric reducing antioxidant power and ferrous ion chelating activity, DPPH radical scavenging activity, and scavenging of hydrogen peroxide assays were also determined. Results of study revealed that phenolic contents were 741,835 and $578 \mu \mathrm{g} / \mathrm{ml}$ of gallic $\mathrm{acid} / 2 \mathrm{~g}$ of coffee, and the total flavonoid contents were 711,802 , and $828 \mu \mathrm{g} / \mathrm{ml}$ of catechin $/ 2 \mathrm{~g}$ of coffee for Nescafé red mug, Turkish coffee and Espresso, respectively. The inhibition percentage of hydrogen peroxide showed highly significant reduction $(\mathrm{p}<0.001)$ in Turkish coffee compared to Nescafé red mug. Further, DPPH activity also showed highly significant reduction $(p<0.01)$ in Espresso and Turkish coffee compared to Nescafé red mug, whereas, Espresso showed a highly significant increase $(\mathrm{p}<0.01)$ in reducing power activity. Regarding the metal chelating activity, Espresso showed a significant decrease $(\mathrm{p}<0.05)$ in metal chelation activity as compared to Nescafé red mug. The current study demonstrated that Nescafé red mug, Turkish coffee and Espresso, were the most consumed coffee types in the Western region of Saudi Arabia according to the survey. These three types of coffee showed high phenolic and flavonoid contents as well as a high antioxidant activity.
\end{abstract}

* Corresponding author

E-mail: haldoghaither@kau.edu.sa (Huda A. Al Doghaither)

Peer review under responsibility of Journal of Experimental Biology and Agricultural Sciences.

Production and Hosting by Horizon Publisher India [HPI] (http://www.horizonpublisherindia.in/).

All rights reserved.
All the article published by Journal of Experimental Biology and Agricultural Sciences is licensed under a Creative Commons Attribution-NonCommercial 4.0 International License Based on a work at www.jebas.org.

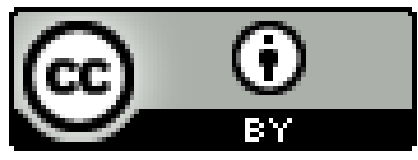




\section{Introduction}

Coffee is one of the most popular beverages all over the world, due to its physiological effects as well as its pleasant taste and aroma (Farah, 2012). It is considered as a functional beverage, primarily because of its high content of antioxidant compounds that exert biological beneficial properties (Dórea \& da Costa, 2005). Polyphenols are very complex group of molecules present in plants (Scalbert et al., 2002; Yardim, 2012). Several compounds can be identified under the term "polyphenols"; these include mainly phenolic and flavonoids acids (Samanidou, 2014). Antioxidants such as polyphenols are important for the maintenance of health and they also provide protection against many diseases such as coronary heart disease and cancer by preventing oxidative stress (Karakaya et al., 2001). Recently, an international interest has been raised on dietary polyphenols for their considerable role in the prevention of various degenerative diseases. This considerable role is based on some epidemiological and clinical studies and on numerous animal studies (Scalbert et al., 2002).

Drinking coffee on regularly basis may prevent cardiovascular diseases and reduce risk of developing type 2 diabetes, Parkinson's disease, stroke and Alzheimer's disease (Butt \& Sultan, 2011). Moreover, coffee has some modest laxative effect also. It can prevent the formation of gallstones, gout and gallbladder diseases by reducing the level of uric acid in blood, which is more significantly observed in men than in women (Dasgupta \& Klein, 2014). From this point of view, the aim of this study was to perform a survey to identify the most consumed types of coffee in Jeddah, Western region of Saudi Arabia, and then estimate the antioxidant activities for these most commonly consumed coffee types identified by this survey.

\section{Materials and Methods}

\subsection{Materials}

Folin-Ciocalteu's phenol reagent and ferric chloride anhydrous were purchased from (BDH, Poole, UK). 2,2-diphenyl-1bicrylhydrazyl, ethanol, trichloroacetic acid (TCA), ferrous chloride, methanol, ferrozine, gallic acid and sodium nitrite were obtained from (Sigma-Aldrich, Poole, UK). Sodium hydroxide pellets were purchased from (PanReacAppliChem, Barcelona, Spain). Aluminum chloride was purchased from (LOBA, Mumbai, India). Ethylenediaminetetraacetic acid (EDTA) was bought from (Fluka, BUCH, Switzerland). Anhydrous sodium carbonate, sodium dihydrogen orthophosphate and di-sodium hydrogen orthophosphate were purchased from (CDH, New Delhi, India). Phosphate buffer saline (PBS) and Hydrogen peroxide were obtained from (Oxoid, Hampshire, UK). Vitamin $\mathrm{C}$ and potassium ferrocyanide were purchased from (FlukaChemika, Buchs, Switzerland). (+)-catechin was purchased from (Steinheim, Germany).

\subsection{Sample preparation}

To estimate the type of coffee that is most commonly consumed, a survey was performed on 900 participants. The survey revealed that the most consumed three types of coffee were Nescafé red mug, Turkish coffee and Espresso. The Nescafé red mug coffee was prepared by adding and mixing $2 \mathrm{~g}$ of Nescafé red mug coffee to $200 \mathrm{ml}$ boiling water. For the Turkish coffee, $3.5 \mathrm{~g}$ of Turkish coffee was added to $75 \mathrm{ml}$ cold water (Karakaya et al., 2001) and mixed well on a heater until the coffee formed small bubbles. For Espresso, $10 \mathrm{~g}$ of coffee was mixed with $180 \mathrm{ml}$ boiling water.

\subsection{Determination of total phenolic content}

The total phenolic contents in coffee samples were determined by following Folin-Ciocalteu method (Przygodzka et al., 2014) for each coffee type. A standard curve was prepared from a serial dilutions of gallic acid (GA) $(0,50,100,150,200,250$ and $500 \mu \mathrm{g} / \mathrm{ml}$ ) and was used to determine the total phenolic contents.

\subsection{Determination of total flavonoid content}

The total flavonoids content of the coffee samples was determined by aluminum chloride colorimetric assay (Dewanto et al., 2002) for all three coffee types. The absorbance of the final product was measured at $510 \mathrm{~nm}$ and the standard curve was prepared from different concentrations of catechin $(0,50$, $100,150,200,250,300$ and $500 \mu \mathrm{g} / \mathrm{ml}$ ).

2.5 Ferric reducing antioxidant power and ferrous ion chelating activity assay

The reducing power of coffee samples was measured at 600 $\mathrm{nm}$ according to the procedure mentioned by Karawita et al. (2005), whereas, the chelating activity of ferrous ion by coffee samples was measured at $562 \mathrm{~nm}$ and was determined by the protocol of Koncic et al. (2011). To determine the ferrous ion chelating percentage, the following equation was used:

$\%$ Chelation $=[(\mathrm{AB}-\mathrm{AA}) / \mathrm{AB}] \times 100$,

Where, AB: absorbance of blank sample and AA: absorbance of sample.

\subsection{DPPH radical scavenging activity assay}

To determine the free radical scavenging activity of coffee samples, 1,1-diphenyl-2-picrylhydrazyl radical (DPPH) assay was used (Ohnishi et al., 1994). The absorbance was recorded at $517 \mathrm{~nm}$ and the inhibition percent equation of DPPH radical scavenging activity was expressed as:

Percentage Scavenging $=[(\mathrm{AB}-\mathrm{AA}) / \mathrm{AB}] \times 100$, 
Where, AB: absorbance of blank sample and

AA: absorbance of sample.

2.7 Scavenging of hydrogen peroxide assay

Hydrogen peroxide scavenging activity was performed according to the procedure of Gülçin et al. (2005) with some modifications. The absorbance was measured at $230 \mathrm{~nm}$ and the percentage of hydrogen peroxide scavenging activity by coffee samples was calculated by the following equation:

Percentage Scavenging $=[(\mathrm{AB}-\mathrm{AA}) / \mathrm{AB}] \times 100$,

Where, AB: absorbance of blank sample and

AA: absorbance of sample.

2.8 Statistical analysis

The statistical analysis was performed using GraphPad Prism version 6.0. The data were analyzed by one-way analysis of variance (ANOVA), followed by a correction with Bonferroni's test. All the results were represented as mean $\pm \mathrm{SD}$ from three independent experiments. Differences between groups were considered to be statistically significant at $p$ value of $<0.05$.

\section{Results}

\subsection{Determination of the most consumed coffee type}

The results obtained from the survey revealed that $55.4 \%$, $23.8 \%$, and $20.7 \%$ of studied participants consumed one, two, and three cups of coffee per day, respectively. Regarding the coffee type, more than 11 coffee types were included in the survey. Among these types, three coffee types showed a high percentage of consumption among the 900 participants. The most common coffee type consumed by the participants was Nescafé red mug coffee $(25.2 \%)$ followed by Turkish coffee (16.4\%) and finally, Espresso (8\%).
3.2 Determination of total phenolic content

As determined by Folin Ciocalteu method, the total phenols content was reported as gallic acid equivalent to standard curve $\left(y=0.0022 x, r^{2}=0.967\right)$. The results for Nescafé red mug, Turkish coffee and Espresso were 741, 835 and $578 \mu \mathrm{g} / \mathrm{ml}$ of gallic acid/2 g of coffee, respectively.

\subsection{Determination of total flavonoid content}

As determined by aluminum chloride method, the total flavonoid content was estimated as catechin equivalent to standard curve $(y=0.0025 x, r 2=0.9213)$. The results of study revealed the presence of 711,802 , and $828 \mu \mathrm{g} / \mathrm{ml}$ of catechin $/ 2$ $\mathrm{g}$ of Nescafé red mug, Turkish coffee and Espresso coffee, respectively. The data revealed that there was no significant difference in the level of total flavonoid between the three coffee types.

3.4 Identification of ferric reducing antioxidant power and ferrous ion chelating activity

The presence of antioxidant, a reluctant substance in the experimental samples caused the reduction of the ferric $\left(\mathrm{Fe}^{3+}\right)$ to the ferrous $\left(\mathrm{Fe}^{2+}\right)$ form. Therefore, by measuring the formation of $\mathrm{Fe}^{2+}$, the reduction reaction can be monitored. In this assay, depending on the reducing power of antioxidant samples, the color of the test solution changed from yellow to green and blue. The reducing power capacity of a compound may serve as a significant signal of its potential antioxidant activity (Gülçin et al., 2010).The ferric reducing antioxidant power of Nescafé red mug, Turkish coffee and Espresso were $0.597,0.428$ and $0.832 \mathrm{mg} / \mathrm{ml}$, respectively. The results showed that Espresso has a significantly $(\mathrm{p}<0.01)$ higher reducing power as compared to the other two coffee samples (figure1).

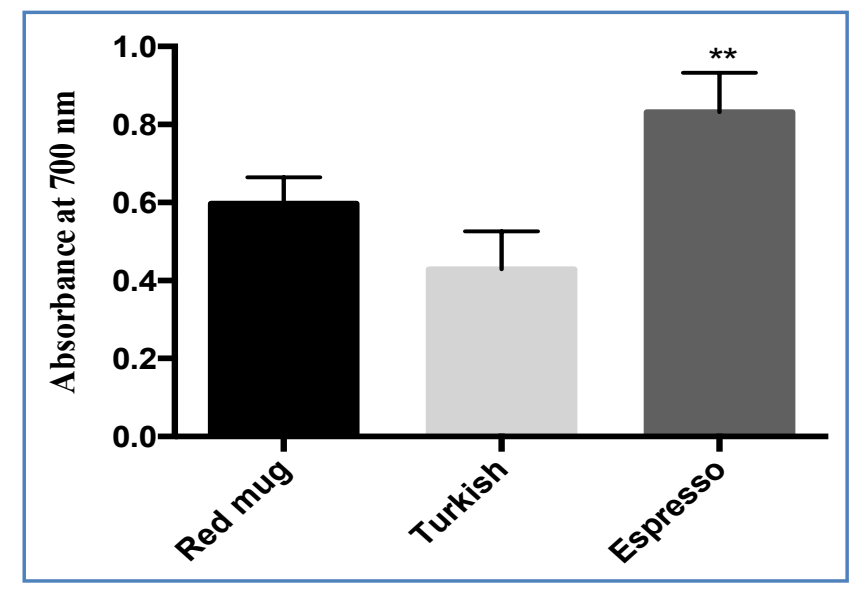

Figure 1 Ferric reducing power of Nescafé red mug, Turkish coffee and Espresso. The values were expressed as means \pm SD $(n>3)$. Comparison of means was calculated using One-way ANOVA followed by Bonferroni's correction test $(* * \mathrm{p}<0.01)$. 


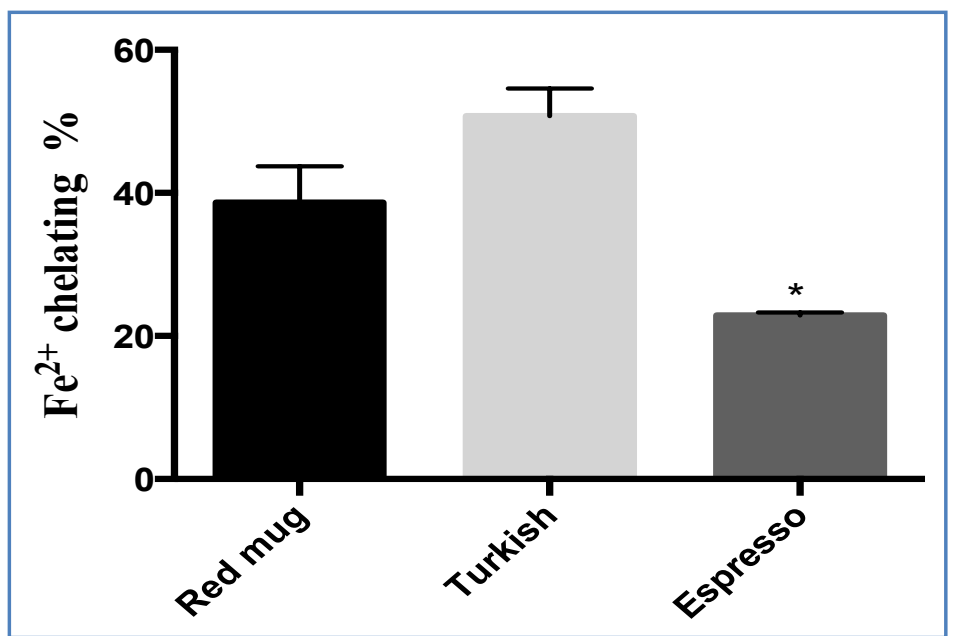

Figure 2 Ferrous ion chelating activity of Nescafé red mug, Turkish coffee and Espresso. Espresso had significantly $(* \mathrm{p}<0.05)$ lower metal-ion chelating activity than Nescafé red mug and Turkish coffee. The values were expressed as means \pm SD ( $>3$ ). Comparison of means was calculated using One-way ANOVA followed by Bonferroni's correction test $\left({ }^{*} \mathrm{p}<0.05\right)$.

On the other hand, the ability of natural plant extracts to chelate transition metal ions can be resulted from the presence of phenolic compounds. These can prevent the formation of metal-induced free radical, by inhibiting the formation of complex between ferrous and ferozine (Yusof et al., 2013). Ferrous ion chelating activity for Nescafé red mug coffee was $38.6 \%$, Turkish coffee was $50.7 \%$ and Espresso was $22.9 \%$. Figure 2 showed that there were no significant differences in $\mathrm{Fe}^{2+}$ chelating activity in Turkish coffee when compared to Nescafé red mug, whereas, Espresso showed a significant decrease in $\mathrm{Fe}^{2+}$ chelating activity when compared to Nescafé red mug.

3.5 Determination of DPPH radical scavenging activity
The DPPH assay is a common method to determine the radical scavenging activity because it is reliable, does not require a special reaction and devices, easy and fast. The free radical scavenging activities of coffee depend on the structural conformation of antioxidant compounds and the ability of these compounds to lose hydrogen. The DPPH free radical, can receive easily an electron or hydrogen from antioxidant molecules to become a stable diamagnetic molecule (Aksoy et al., 2013).The DPPH radical scavenging percent of Nescafé red mug, Turkish coffee and Espresso were $45.6 \%, 28.7 \%$ and $23.6 \%$, respectively. The results showed that there were significant decreases $(\mathrm{p}<0.01)$ in scavenging activity in Turkish coffee and Espresso compared to Nescafé red mug (figure 3).

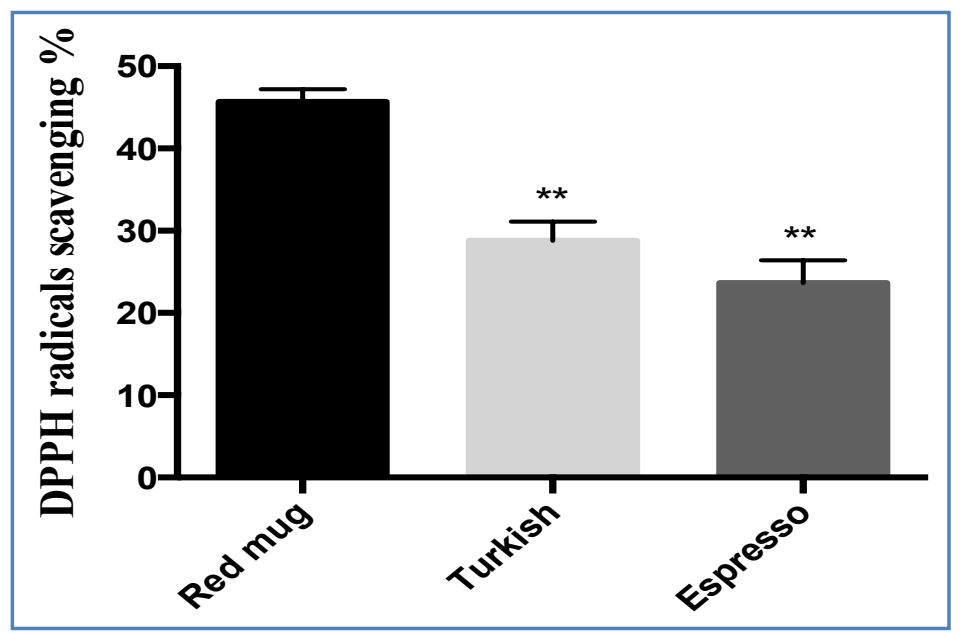

Figure 3 DPPH radical scavenging activity of Nescafé red mug, Turkish coffee and Espresso. Turkish coffee and Espresso have significantly $(\mathrm{p}<0.01)$ lower radical scavenging activity compared to Nescafé red mug. Comparison of means was calculated using Oneway ANOVA followed by Bonferroni's correction test $(* * \mathrm{p}<0.01)$. 


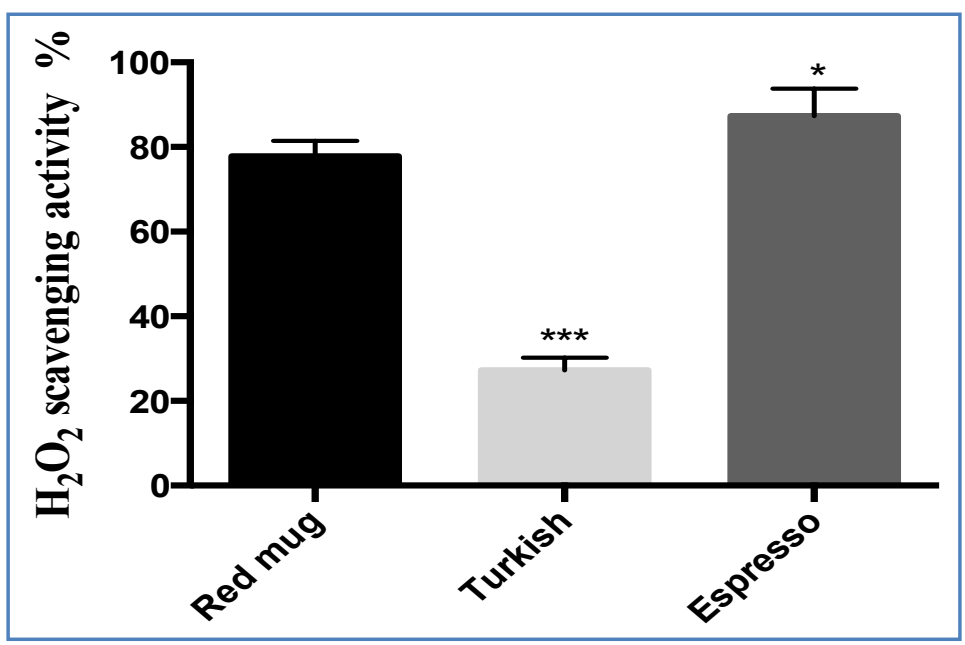

Figure 4 Hydrogen peroxide scavenging activity of Nescafé Red mug, Turkish coffee and Espresso. Turkish coffee had significantly lower $(* * * p<0.001)$ scavenging activity for hydrogen peroxide, whereas Espresso had significantly higher $(* \mathrm{p}<0.05)$ scavenging activity for hydrogen peroxide compared to Nescafe red mug. Comparison of means was calculated using One-way ANOVA followed by Bonferroni's correction test $(* \mathrm{p}<0.05$ and $* * * \mathrm{p}<0.001)$.

3.6 Determination of hydrogen peroxide scavenging activity

Reactive oxygen species (ROS) include non-radical reactive derivatives and free radicals. Reactivity of non-radical species is generally weaker than free radicals. Hydrogen peroxide $\left(\mathrm{H}_{2} \mathrm{O}_{2}\right)$ is an example of non- radical derivatives. Free radicals are unstable because they contain one or more electrons unpaired in their outer shell. Therefore, they tend to become stable either by accepting or by donating an electron (Priyanka et al., 2013). The scavenging percent of hydrogen peroxide of Nescafé red mug, Turkish coffee and Espresso were $77.8 \%$, $27.2 \%$ and $87.2 \%$, respectively, indicating that Turkish coffee had a highly significant reduction $(\mathrm{p}<0.001)$ in $\mathrm{H}_{2} \mathrm{O}_{2}$ scavenging activity compared to the two other tested coffee types (figure 4).

\section{Discussion}

In the last decade, there has been a lot of attention to polyphenols, owing to their antioxidant capacity, metal chelating and free radical scavenging, and their possible beneficial effects on human health (Wollgast \& Anklam, 2000). In plants, most of the antioxidant potential is due to the redox properties of phenolic compounds, which act as hydrogen donors, singlet oxygen quenchers and reducing agents (Kasote et al., 2015). Through various mechanisms, antioxidant activity of polyphenols may act as reducing, chelating and scavenging agent; act as cofactors of enzymes catalyzing oxidative reactions, terminate radical chain reactions, inhibit oxidases, and stabilize free radicals (Bogucka-Kocka et al., 2016). Maillard reaction is responsible for the generation of compounds called melanoidins, brown pigments in roasted coffee, from reducing amino acids and proteins or sugars during food processing and preservation (Wang et al., 2011; Karamac et al., 2005). Thermal processing can induce the formation of new compounds with antioxidant properties or improve the properties of naturally occurring antioxidant. Therefore, the overall antioxidant activity remains unchanged or increased (Somporn et al., 2011).

Moreover, the antioxidant activity of coffee is related to the presence of important compounds such as chlorogenic, caffeic, ferulic, and n-coumaric acids. In some researches, caffeine was considered to be an important antioxidant agent (Karamac et al., 2005). According to the conducted survey, the results of the current study showed that Nescafé red mug, Turkish coffee and Espresso were the most consumed coffee types in Jeddah city, Western region of Saudi Arabia. Furthermore, there were no significant differences in reducing power activity between Nescafé red mug and Turkish coffee while Espresso had a higher ferric reducing power activity. This higher activity of Espresso to reduce ferric ion might be due to the high chlorogenic acid content. Indeed, a previous study supported a strong relationship between ferric reducing power activity and chlorogenic acid content (Moreira et al., 2005).

Regarding the scavenging activity, results of present study revealed that the highest radical scavenging activity was in Nescafé red mug and it was followed by Turkish coffee, whereas, the lowest scavenging activity was found in Espresso. Reduction in radical scavenging activity might be due to the roasting degree, and as well known the antiradical efficiency can be degraded during roasting (Erdem et al., 2016). Indeed, previous researches have reported that roasting degree can influence the total phenolic content and radical scavenging activity of coffee beans. The dark roasted coffee beans had lower radical scavenging than light roasted coffee beans (Del Castillo et al., 2002; Shan et al., 2015). Moreover, results of study revealed that the DPPH radical scavenging activity express lowest among the other types. It was previously 
reported that the concentrations of caffeic acid and phydroxybenzoic acid seemed to be high in medium roasting coffee such as Nescafé red mug and Turkish coffee. In DPPH radical scavenging assay, hydroxybenzoic acid acts as a weak scavenger of DPPH radical (Somporn et al., 2011). Furthermore, results from hydrogen peroxide scavenging experiments showed a significant increase in both Espresso and in Nescafé red mug and a significant decrease in the Turkish coffee sample. The concentrations of ferulic acid, vanillic acid and protocatechuic acid, tend to increase when roasted in higher degrees such as Espresso (Shan et al., 2015). Vanillic acid is considered as one of the most active components in scavenging hydrogen peroxide. Hydroxybenzoic acid, which is present in Turkish coffee, showed a moderate antioxidant scavenging activity of hydrogen peroxide. Hydroxybenzoic acid is a weak scavenger of hydrogen peroxide (Somporn et al., 2011). Thus, better peroxyl radical scavenging activity is exerted in roasted dark coffee than the less roasted ones (Sánchez-González et al., 2005).

In addition, in this study, Nescafé red mug and Turkish coffee showed a higher chelating activity percentage than Espresso. hydroxycinnamic acid such as caffeic acid, which is high in Nescafé red mug and Turkish coffee, showed a better complex formation than protocatechuic acid, which is higher in Espresso coffee. The capacity of complex formation by hydroxycinnamic acid is relatively better than protocatechuic acid. Complex formation does not occur by all phenolic compounds, some of them are not metal chelators. For instance, phenolic acids, which are bearing catechol or gollyol group, such a vanillic acid and ferulicacid, which are present in high percentage in Espresso coffee, do not form chelation complex. On the other hand, phenolic acids, which are bearing catechol or gallyol group such as caffeic acid, showed a strong chelation activity (Andjelkovic et al., 2006).

\section{Conclusion}

This study demonstrated that Nescafé red mug, Turkish coffee and Espresso, were the most consumed coffee types in Jeddah according to the conducted survey. These three types of coffee showed high phenolic contents as well as a high antioxidant activity. Further in vivo and in vitro researches should be performed to estimate the benefits of these coffee brews and their effects on human health.

\section{Acknowledgment}

The authors would like to express their thanks for the Science Research and Innovation Unit at the Faculty of Sciences, King Abdulaziz University, Jeddah, Saudi Arabia, for supporting this work financially.

\section{Conflict of interest}

Authors would hereby like to declare that there is no conflict of interests that could possibly arise.

\section{References}

Aksoy L, Kolay E, Aĝilönü Y, Aslan Z, Mustafa Kargioglu (2013) Free radical scavenging activity, total phenolic content, total antioxidant status, and total oxidant status of endemic Thermopsis turcica. Saudi Journal of Biological Sciences20: 235-239. DOI: http://dx.doi.org/10.1016/j.sjbs.2013.02.003.

Andjelkovic M, Van Camp J, De Meulenaer B, Depaemelaere G, Socaciu C,Verloo M, Verhe R (2006) Iron-chelation properties of phenolic acids bearing catechol and galloyl groups. Food Chemistry 98: 23-31. DOI: http://dx.doi.org/10.1016/j.foodchem.2005.05.044.

Bogucka-Kocka A, Zidorn C, Kasprzycka M, Szymczak G, SzewczykK (2016) Phenolic acid content, antioxidant and cytotoxic activities of four kalanchoë species. Saudi Journal of Biological Sciences. DOI: http://dx.doi.org/10.1016/j.sjbs.2016.01.037.

Butt MS, Sultan MT (2011) Coffee and its consumption: benefits and risks. Critical Reviews in Food Science and Nutrition 51: 363-373. DOI: 10.1080/10408390903586412.

Dasgupta A, Klein K (2014) Tea, coffee, and chocolate. In: Antioxidants in food, vitamins and supplements : Prevention and treatment of disease,Elsevier, Amsterdam, Netherlands publication. DOI: http://dx.doi.org/10.1016/B978-0-12405872-9.00013-6

Del Castillo MD, Ames JM, Gordon MH (2002) Effect of roasting on the antioxidant activity of coffee brews. Journal of Agricultural and Food Chemistry 50: 3698-3703. DOI: 10.1021/jf011702q.

Dewanto V, Wu X, Adom KK, Liu RH (2002) Thermal processing enhances the nutritional value of tomatoes by increasing total antioxidant activity thermal processing enhances the nutritional value of tomatoes by increasing total antioxidant activity. Journal of Agricultural and Food Chemistry 50: 3010-3014. DOI: 10.1021/jf0115589.

Dórea JG, da Costa, THM (2005) Is coffee a functional food?. British Journal of Nutrition 93: 773-782. DOI: 10.1079/BJN20051370.

Erdem S, Senol F, Budakoglu E, Orhan I, Sener B (2016) Exploring in vitro neurobiological effects and high-pressure liquid chromatography-assisted quantitation of chlorogenic acid in 18 turkish coffee brands. Journal of Food and Drug Analysis 24: $112-120 . \quad$ DOI: http://dx.doi.org/10.1016/j.jfda.2015.08.001.

Farah A (2012) Coffee constituents. In: Chu YF (Ed).Coffee:Emerging Health Effects and Disease Prevention, JohnWiley, New Jersey, United States. 
Gülçin Ilhami, Alici H, Cesur M (2005) Determination of in vitro antioxidant and radical scavenging activities of propofol. Chemical \& Pharmaceutical Bulletin 53: 281-285. DOI: http://doi.org/10.1248/cpb.53.281.

Gülçin I, Huyut Z, Elmastas M, Aboul-Enein HY (2010) Radical scavenging and antioxidant activity of tannic acid. Arabian Journal of Chemistry 3: 43-53. DOI: http://dx.doi.org/10.1016/j.arabjc.2009.12.008.

Karakaya S, El SN, Tas A (2001) Antioxidant activity of some foods containing phenolic compounds. International Journal of Food Sciences and Nutrition 52: 501-508.

Karamac M, Bucinski A, Pegg R, Amarowicz R (2005) Antioxidant and antiradical activity of coffee. Czech Journal of Food Sciences 23: 64-68.

Karawita R, Siriwardhana N, Lee K, Heo M, Yeo I, Lee Y, Jeon Y (2005) Reactive oxygen species scavenging, metal chelation, reducing power and lipid peroxidation inhibition properties of different solvent fractions from hizikia fusiformis. European Food Research and Technology 220: 363-371. doi:10.1007/s00217-004-1044-9.

Kasote DM, Katyare SS, Hegde MV, Bae H (2015) Significance of antioxidant potential of plants and its relevance to therapeutic applications. International Journal of Biological Sciences 11: 982-991. doi:10.7150/ijbs.12096.

Koncic MZ, Barbaric M, Perkovic I, Zorc B (2011) Antiradical, chelating and antioxidant activities of hydroxamic acids and hydroxyureas. Molecules 16:6232-6242. doi: 10.3390/molecules 16086232 .

Moreira DP, Monteiro MC, Ribeiro-Alves M, Donangelo CM, Trugo LC (2005) Contribution of chlorogenic acids to the ironreducing activity of coffee beverages. Journal of Agricultural and Food Chemistry 53: 1399-1402. DOI: 10.1021/jf0485436.

Ohnishi M, Morishita H, Iwahashi H, Toda S, Shirataki Y, Kimura M, Kido R (1994) Inhibitory effects of chlorogenic acids on linoleic-acid peroxidation and hemolysis. Phytochemistry 36:579-583. DOI: http://dx.doi.org/10.1016/S0031-9422(00)89778-2

Priyanka B, Anitha K, Shirisha K, Sk J, Dipankar B, Rajesh K (2013) Evaluation of antioxidant activity of ethanolic root extract of Albizia lebbeck (L.) benth. International Research Journal of Pharmaceutical and Applied Sciences 3:93-101.

Przygodzka M, Zielinska D, Ciesarová Z, Kukurová K, Zielinski H (2014) Comparison of methods for evaluation of the antioxidant capacity and phenolic compounds in common spices. LWT - Food Science and Technology 58:321-326. DOI: http://dx.doi.org/10.1016/j.lwt.2013.09.019

Samanidou V (2014) Determination of polyphenols and major purine alkaloids in coffee: an overview. A (Victor R. Preedy). Coffee in Health and Disease Prevention. Elsevier,Amsterdam, Netherlands.

Sánchez-González I, Jiménez-Escrig A, Saura-Calixto F (2005) In vitro antioxidant activity of coffees brewed using different procedures (italian, espresso and filter). Food Chemistry 90: $133-139 . \quad$ DOI: http://dx.doi.org/10.1016/j.foodchem.2004.03.037.

Scalbert A, Morand C, Manach C, Rémésy C (2002) Absorption and metabolism of polyphenols in the gut and impact on health. Biomedicine and Pharmacotherapy 56: 276282. DOI: http://dx.doi.org/10.1016/S0753-3322(02)00205-6.

Shan OE, Zzaman W, Yang TA (2015) Effect of superheated steam roasting on radical scavenging activity and phenolic content of robusta coffee beans. International Journal on Advanced Science, Engineering and Information Technology 5: 99-102. DOI: http://dx.doi.org/10.18517/ijaseit.5.2.493

Somporn C, Kamtuo A, Theerakulpisut P, Siriamornpun S (2011) Effects of roasting degree on radical scavenging activity, phenolics and volatile compounds of arabica coffee beans (Coffea arabica L. cv. catimor). International Journal of Food Science and Technology 46: 2287-2296. DOI: 10.1111/j.1365-2621.2011.02748.x.

Wang H, Qian H, Yao W (2011) Melanoidins produced by the maillard reaction: Structure and biological activity. Food Chemistry128: $573-584$ http://dx.doi.org/10.1016/j.foodchem.2011.03.075.

Wollgast J, Anklam E (2000) Polyphenols in chocolate: Is there a contribution to human health? Food Research International33: 449-459. DOI: http://dx.doi.org/10.1016/S0963-9969(00)00069-7.

Yardim Y (2012) Electrochemical behavior of chlorogenic acid at a boron-doped diamond electrode and estimation of the antioxidant capacity in the coffee samples based on its oxidation peak. Journal of Food Science 77: 408-413. DOI: 10.1111/j.1750-3841.2011.02609.x.

Yusof N, Hasan M, Armayni U, Bin M, Mohsin H, Wahab I (2013) The ferrous ion chelating assay of pandanus extracts. The Open Conference Proccedings Journal 4:155. 